\title{
Case Report \\ Rapidly Evolving Coronary Aneurysm in a Patient with Rheumatoid Arthritis
}

\section{Reji Pappy, Siddharth Wayangankar, Thomachan Kalapura, and Mazen S. Abu-Fadel}

Section of Cardiovascular Medicine, Department of Medicine, University of Oklahoma Health Sciences Center, 920 S. L. Young Boulevard., WP 3010 Oklahoma City, OK 73104, USA

Correspondence should be addressed to Mazen S. Abu-Fadel, mazen-abufadel@ouhsc.edu

Received 1 December 2010; Revised 31 December 2010; Accepted 11 January 2011

Academic Editor: Marco Metra

Copyright (๑) 2011 Reji Pappy et al. This is an open access article distributed under the Creative Commons Attribution License, which permits unrestricted use, distribution, and reproduction in any medium, provided the original work is properly cited.

Coronary artery aneurysm (CAA) formation in the setting of an acute inflammatory state due to connective tissue disease is rare. We report a case of rapid progression from an ectatic to an aneursymatic left circumflex coronary artery leading to an acute coronary event in a patient with rheumatoid arthritis (RA). We report the accelerated growth of the aneurysm as it was temporally related to the lapse in treatment and the management strategies involved with this entity.

\section{Introduction}

Coronary artery aneurysm (CAA) formation in the setting of systemic inflammatory conditions has been described. We report a unique case of rapid development from ectasia to aneurysm of the left circumflex coronary artery after a lapse in treatment for rheumatoid arthritis (RA), which was complicated by a non-ST elevation myocardial infarction (NSTEMI). We describe our management strategy and also discuss other systemic immune-inflammatory conditions that have been associated with CAAs.

\section{Case}

A 67-year-old Caucasian man with a past medical history of severe seropositive RA, hypertension, coronary artery disease (CAD) with previous stents, and ectasia of all three coronary arteries presented with chest pain, nausea, and diaphoresis that has been ongoing for the past 3-4 days. He reported self-discontinuation of immunosuppressive therapy for RA approximately four months ago, and subsequently he has been experiencing pain in multiple joints and was found to have elevated inflammatory markers (rheumatoid factor). Upon further workup, he was found to have severe active synovitis of the left wrist and left knee. The outpatient medications included aspirin, clopidogrel, and warfarin which were prescribed to prevent thrombus formation in the ectatic coronaries. However, due to poor adherence to this medical therapy, the admission international normalized ratio (INR) was found to be subtherapeutic. Elevated cardiac biomarkers and electrocardiographic (ECG) changes (ST depression in leads V5-V6) were consistent with an NSTEMI, and it was decided to proceed with coronary angiography. This revealed a large, 23 millimeter mid-left circumflex coronary artery aneurysm complicated by subtotal thrombosis with distal run-off occlusion (Figure 1). The diffuse coronary artery ectasia in the other coronary arteries appeared to be stable. The aneurysm was determined to be rapidly enlarging, as this was not present on a previous coronary angiogram six months prior (Figure 2). A left ventriculogram revealed an ejection fraction of $40 \%$.

Attempts to cross the aneurysm to the distal artery with multiple wires and catheters were unsuccessful due to extensive clot burden in the aneurysm. After consulting with the cardiac surgeons, it was decided to proceed with coil embolization of this aneurysm to prevent clot propagation and potential rupture in the future. A 7 French Cordis XB4 guiding catheter (Cordis Corp., Bridgewater, NJ) was used to engage the left circumflex coronary artery. A 0.014 inch J-tip guidewire was used to position a 0.018 inch inner diameter microcatheter to deliver one 6-Nester (Cook Medical, Bloomington, IN) and one 2-6 Tornado coil occluders 


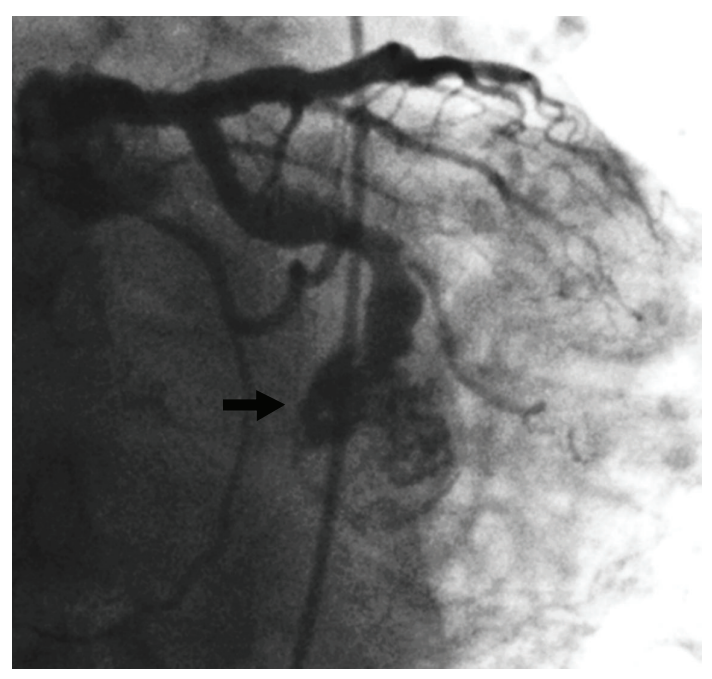

FIGURE 1: Subtotal thrombus occlusion of the left circumflex coronary artery aneurysm with blood flow into the aneurysm (arrow).

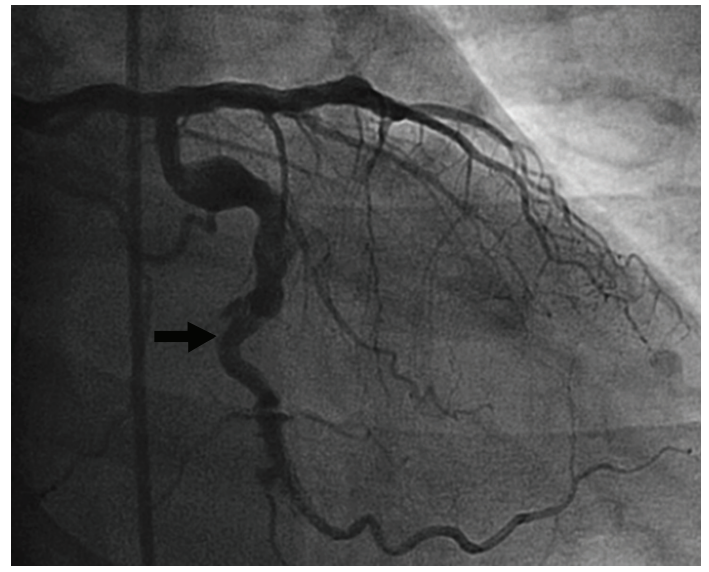

Figure 2: Angiogram six months prior reveals a mildly ectatic but not aneurysmatic left circumflex coronary artery (arrow).

(Cook Medical) into the afferent limb of the aneurysm. Postdeployment images demonstrated complete occlusion of the aneurysm (Figure 3 ). The patient reported mild chest pain during the procedure which resolved spontaneously, and postprocedure cardiac enzymes were down-trending compared to initial values. Based on clinical, laboratory, and angiographic data, it was postulated that the patient may have experienced RA-induced coronary vasculitis leading to aneurysm formation therefore, the patient was initiated on immunosuppressive therapy (prednisone and methotrexate) for RA. He was discharged home within 48 hours and at 1 -year clinical followup, the patient reports no symptoms and has excellent functional capacity with a left ventricular ejection fraction of $50 \%$ with no wall motion abnormalities.

\section{Discussion}

Although the vast majority of acute coronary events result from atherosclerotic lesions in coronary arteries, some cases

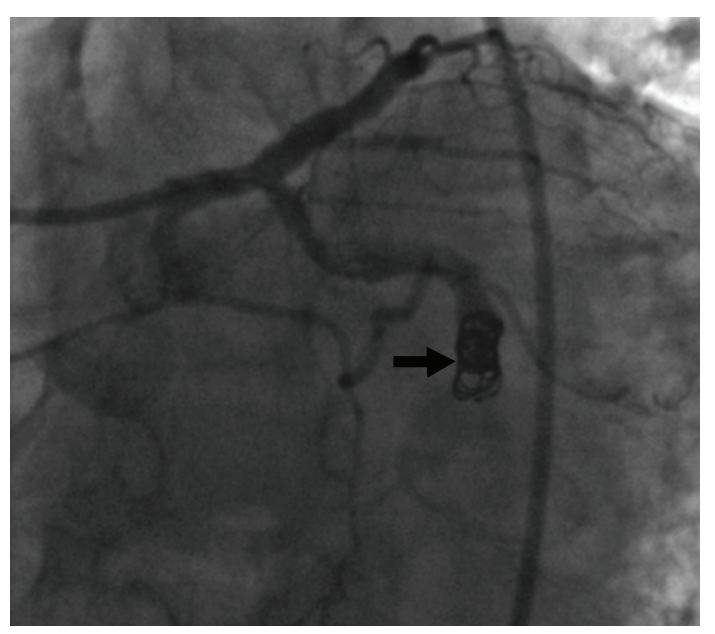

FIGURE 3: Successful coiling with no blood flow into the aneurysm (arrow).

of myocardial infarction are related to nonatherosclerotic processes, such as coronary embolism, vasospasm, dissection, congenital abnormalities, and, in our case, aneurysm. CAAs are rare but potentially fatal if it is not managed in a judicious and timely manner. The first pathologic description of a CAA was made by Morgagni in 1761 [1]. Munker and colleagues diagnosed this by angiography in 1958 [2]. The wide range in the reported incidence $(0.15 \%$ to $4.9 \%$ ) is attributed to the conceptual differences in the definition of coronary ectasia and aneurysm $[1,2]$. CAA is defined as a segmental dilatation of the vessel, with a diameter $\geq 1.5$ times (some authors have defined it as $\geq 2$ times) of an adjacent normal section. Left main coronary artery aneurysm is defined as segmental dilatation $\geq 1.5$ times the diameter of the widest normal coronary artery. Ectasia is characterized by a more diffuse dilatation involving at least $50 \%$ of the vessel length. Coronary ectasia and aneurysm are thought to represent subsequent stages of a dilatative process [3].

There are many inflammatory disorders, connective tissue disorders, and infectious etiologies that have been associated with CAAs. Atherosclerosis is the most common etiology in the U.S., and Kawasaki's disease is the most common etiology in the Far East [4]. The pathophysiology of the inflammatory conditions involve coronary vasculitis, which causes thickening of the arteries leading to their occlusion, and in some cases, weakening of the medial layer of the vessel wall, leading to aneurysms. CAAs have a male predominance and can be focal or diffuse in nature, and classified morphologically as saccular or fusiform. The most common location of CAAs, in order of decreasing frequency, are the right coronary artery $(40-87 \%)$, left anterior descending artery, left circumflex artery, and then rarely the left main coronary artery [5].

Coronary artery aneurysm associated with RA is rare but has been described [6]. RA is a systemic inflammatory disease characterized by chronic and erosive synovitis that involves peripheral joints. A variety of extra-articular 
manifestations have been described in RA and include subcutaneous nodules, episcleritis, mononeuritis multiplex, serositis, petechiae, and skin ulcers. Moreover, RA has been associated with cardiovascular manifestations which include pericarditis, cardiomyopathy, cardiac amyloidosis, coronary vasculitis, arrhythmia, valvulopathy, congestive heart failure, and ischemic heart disease. Systemic vasculitis complicating RA has a reported mortality rate of up to 30\% [7]. RAinduced vasculitis has been implicated in causing aneurysms of the aorta and other vascular beds, that is, the renal, vertebral, spinal, hepatic, and coronary vasculature. Moreover, the proinflammatory state of RA can also lead to accelerated atherosclerosis, and it is a well-known independent risk factor for multivessel CAD accounting for the increased prevalence of coronary heart disease in this group of patients [8]. However, the challenge for proponents of the "coronary vasculitis" theory is clinically determining the etiology of coronary changes (ectasia, aneurysms) as being primarily due to vasculitis or atherosclerosis or the combination of both. RA involvement in CAAs is primarily a clinical diagnosis, as the definitive diagnosis is usually established at autopsy.

Overall, most patients with CAAs are asymptomatic; however, symptoms related to thrombus formation with subsequent distal coronary thromboembolism, extrinsic native vessel compression, fistula formation, coronary spasm, or even rupture can occur. Medical, interventional and surgical strategies to manage coronary aneurysms have been described. Preventative therapy for thrombosis in the setting of CAA includes the use of single or dual antiplatelet therapy, with or without the addition of antivitamin $\mathrm{K}$ therapy. Use of antiplatelet or anticoagulation therapy in patients with CAAs is based on anecdotal reports. Percutaneous strategies include coil embolization, autologous saphenous vein-covered stent grafting, polytetrafluoroethylene- (PTFE)covered stent grafts, and one case report of DES implantation superimposed on a PTFE-covered stent graft [9, 10]. Surgical strategies that have been described include aneurysm ligation, resection, marsupialization with interposition graft, and coronary artery bypass surgery. The majority of the experience regarding the aforementioned strategies stem from atherosclerosis-induced CAAs. However, special consideration needs to be taken when an immuneinflammatory condition is involved. Studies with systemic inflammatory diseases, that is, Takaysu's arteritis (TA) have revealed that the weakening of the walls of the affected vessels, as well as the increased risk of thrombosis, make invasive procedures problematic and can result in a high rate of restenosis and immediate elastic recoil $[11,12]$. Moreover, several studies have shown an increased risk of failure of surgical revascularization in patients with TA [13]. Treatment strategies to improve clinical outcomes in vasculitis patients with CAAs have mostly been described in Takayasu's arteritis, Kawasaki's disease, Wegener's granulomatosis, polyarteritis nodosa, and giant cell arteritis either as case reports or reviews [14-16]. However, a rapid progression of coronary ectasia to an aneurysm has not been previously described in RA, but has been reported in a patient with Takayasu's arteritis [17]. Treatment strategies to improve outcomes in vasculitis-induced CAA involve the use of immunosuppressive therapy to abate the underlying inflammatory process. The role of catheter-based intervention needs to be further explored in this particular patient population. It must be emphasized that there is no consensus on how coronary lesions related to systemic inflammatory diseases should be treated.

Our patient presented as a case of RA flare-up due to a lapse in treatment. This not only caused elevation of inflammatory markers and severe synovitis but probably led to rapid coronary artery aneurysm formation likely due to vasculitis. In our patient, both traditional risk factors of atherosclerosis and the immune-inflammatory state of RA likely contributed to chronic coronary artery ectasia. Coronary vessels with ectatic areas are more prone to aneurysmatic growth than normal vessel segments. We postulate that vasculitis superimposed on a chronically weak vessel wall led to rapid aneurysm formation. The CAA predisposed to stasis and then thrombus formation. Eventually, an acute thrombus superimposed on a chronic one lead to an NSTEMI. Perhaps, adherence to warfarin may have prevented the acute coronary event; however, continued expansion of the aneurysm could have led to potential rupture.

Our decision to proceed with percutaneous management was predicated on the observance of a rapidly expanding aneurysm that was not present six months previously. The combination of both atherosclerosis and RA deemed this aneurysm at high risk for further growth and potential rupture; therefore, it was decided to proceed with both coiling and aggressive medical management of RA. The presence of thrombus in the aneurysm prevented wiring of the lesion into the distal lumen. Moreover, this also precluded deployment of a covered stent, thrombus aspiration, and other interventional strategies. The patient was discharged on a medical regimen consisting of dual antiplatelet therapy, antithrombotic therapy, and immunosuppressive agents (methotrexate and prednisone). Data suggest that methotrexate-treated RA patients have reductions in cardiovascular morbidity and mortality [18]. There are no guideline recommendations for the management of CAAs due to the limited data; therefore, the management strategy should be individualized based on aneurysm size, location, pathophysiology, and patient symptoms.

\section{Conclusion}

Coronary artery aneurysms have a causal association with $\mathrm{RA}$. The rate of CAA expansion and rupture is unpredictable; therefore, prompt initiation of immunosuppressive therapy when indicated is crucial. Each patient presents with unique clinical and angiographic features, so the treatment strategy that is utilized should be formulated as such. As the mechanisms underlying the formation and growth of CAAs are elucidated, guidelines on how to screen and manage this condition will be forthcoming. Currently, the challenge remains to differentiate high-risk CAAs versus those associated with a benign outcome. 


\section{Disclosure}

All four authors of this paper have no conflicts of interest to disclose.

\section{References}

[1] J. B. Morgagni, "De sedibus et causis morborum," Venectus Tom I, Epis, vol. 27, article 28, 1761.

[2] T. M. Munker, O. Peterson, and J. Vesterdal, "Congential aneurysm of the coronary artery with an arteriovenous fistula," Acta Radiologica, vol. 50, pp. 333-336, 1958.

[3] M. Syed and M. Lesch, "Coronary artery aneurysm: a review," Progress in Cardiovascular Diseases, vol. 40, no. 1, pp. 77-84, 1997.

[4] P. Cohen and P. T. Ogara, "Coronary artery aneurysms: a review of the natural history, pathophysiology, and management," Cardiology in Review, vol. 16, no. 6, pp. 301-304, 2008.

[5] G. G. Hartnell, B. M. Parnell, and R. B. Pridie, "Coronary artery ectasia: its prevalence and clinical significance in 4993 patients," British Heart Journal, vol. 54, no. 4, pp. 392-395, 1985.

[6] K. Y. Wang, C. T. Ting, M. ST. J. Sutton, and Y. T. Chen, "Coronary artery aneurysms: a 25-patient study," Catheterization and Cardiovascular Interventions, vol. 48, no. 1, pp. 31-38, 1999.

[7] G. A. V. Albada-Kuipers, J. A. Bruijn, M. L. Westedt, F. C. Breedveld, and F. Eulderink, "Coronary arteritis complication rheumatoid arthritis," Annals of the Rheumatic Diseases, vol. 45, pp. 963-965, 1986.

[8] M. Boers, B. Dijkmans, S. Gabriel, H. Maradit-Kremers, J. O'Dell, and T. Pincus, "Making an impact on mortality in rheumatoid arthritis: targeting cardiovascular comorbidity," Arthritis and Rheumatism, vol. 50, no. 6, pp. 1734-1739, 2004.

[9] R. K. Ghanta, S. Paul, and G. S. Couper, "Successful revascularization of multiple coronary artery aneurysms using a combination of surgical strategies," Annals of Thoracic Surgery, vol. 84, no. 2, pp. e10-e11, 2007.

[10] T. Suselbeck, D. Haghi, M. Borggrefe, and J. J. Kaden, "Percutaneous treatment of a coronary aneurysm by stent graft and drug-eluting stent implantation: a potential method to reduce stent graft restenosis," Journal of Interventional Cardiology, vol. 21, no. 4, pp. 325-328, 2008.

[11] J. W. Son, K. K. Koh, Q. Dang, I. S. Choi, and E. K. Shin, "Recurrent restenosis following stent and rotational atherectomy of coronary artery stenosis in Takayasu's arteritis," International Journal of Cardiology, vol. 65, no. 3, pp. 295-300, 1998.

[12] I. Antelmi, L. Magalhaes, B. Caramelli et al., "Rescue PTCA in a 16 year old boy with Takayasu's disease and evolving myocardial infarciton," Arquivos Brasileiros de Cardiologia, vol. 60, pp. 37-38, 1993.

[13] K. S. Stamatelopoulos, G. D. Kitas, C. M. Papamichael et al., "Atherosclerosis in rheumatoid arthritis versus diabetes: a comparative study," Arteriosclerosis, Thrombosis, and Vascular Biology, vol. 29, no. 10, pp. 1702-1708, 2009.

[14] J. T. Lie, "Pathology of isolated nonclassical and catastrophic manifestations of Takayasu arteritis," International Journal of Cardiology, vol. 66, no. 1, supplement, pp. S11-S21, 1998.

[15] G. Schmidt, R. Gareis, and T. Störk, "Direct percutaneous coronary intervention for NSTEMI in a patient with seropositive Wegener's granulomatosis," Zeitschrift fur Kardiologie, vol. 94, no. 9, pp. 583-587, 2005.
[16] O. Amir, B. Kar, A. B. Civitello, N. Palanichamy, A. Shakir, and R. M. Delgado, "Unprotected left main stent placement in a patient with Takayasu's arteritis: an unusual solution for an unusual disease," Texas Heart Institute Journal, vol. 33, no. 2, pp. 253-255, 2006.

[17] A. Araszkiewicz, M. Prech, P. Hrycaj, M. Lesiak, S. Grajek, and A. Cieslinski, "Acute myocardial infarction and rapid development of coronary aneurysms in a young womanunusual presentation of Takayasu arteritis?" Canadian Journal of Cardiology, vol. 23, no. 1, pp. 61-63, 2007.

[18] P. M. Ridker, "Testing the inflammatory hypothesis of atherothrombosis: scientific rationale for the cardiovascular inflammation reduction trial (CIRT)," Journal of Thrombosis and Haemostasis, vol. 7, no. 1, supplement, pp. 332-339, 2009. 


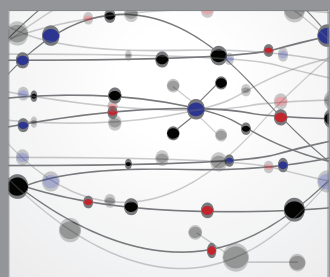

The Scientific World Journal
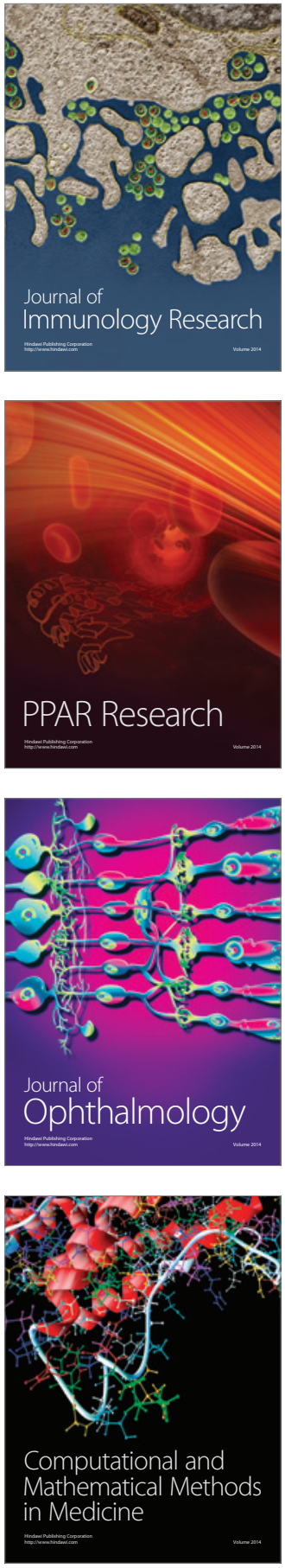

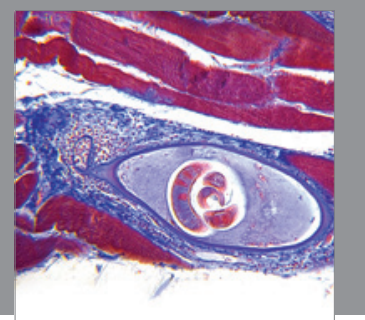

Gastroenterology

Research and Practice
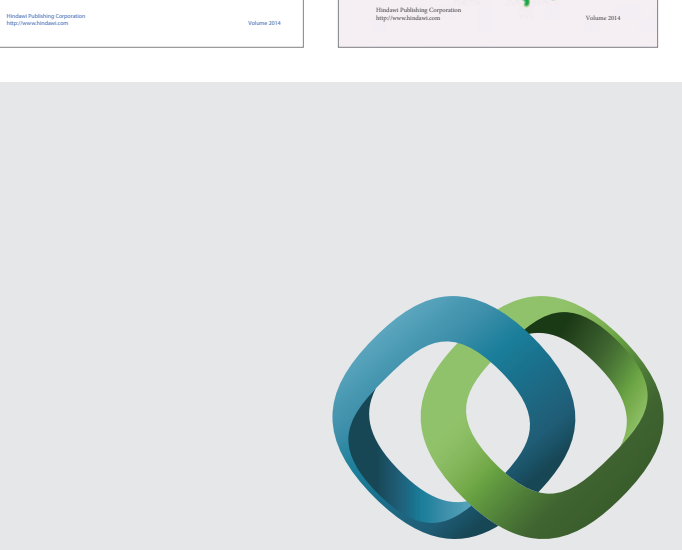

\section{Hindawi}

Submit your manuscripts at

http://www.hindawi.com
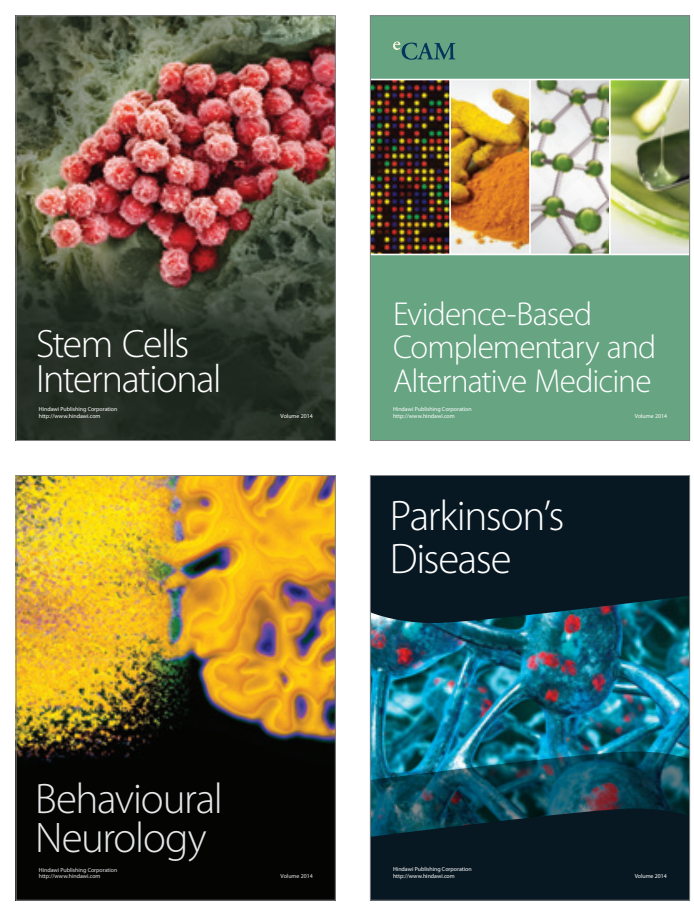

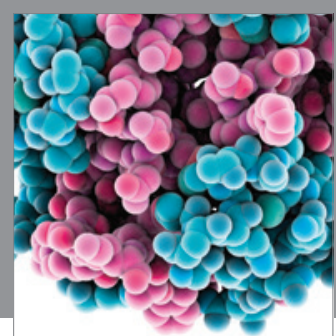

Journal of
Diabetes Research

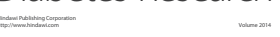

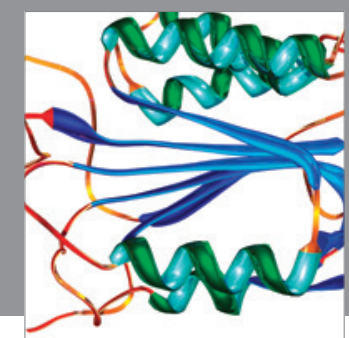

Disease Markers
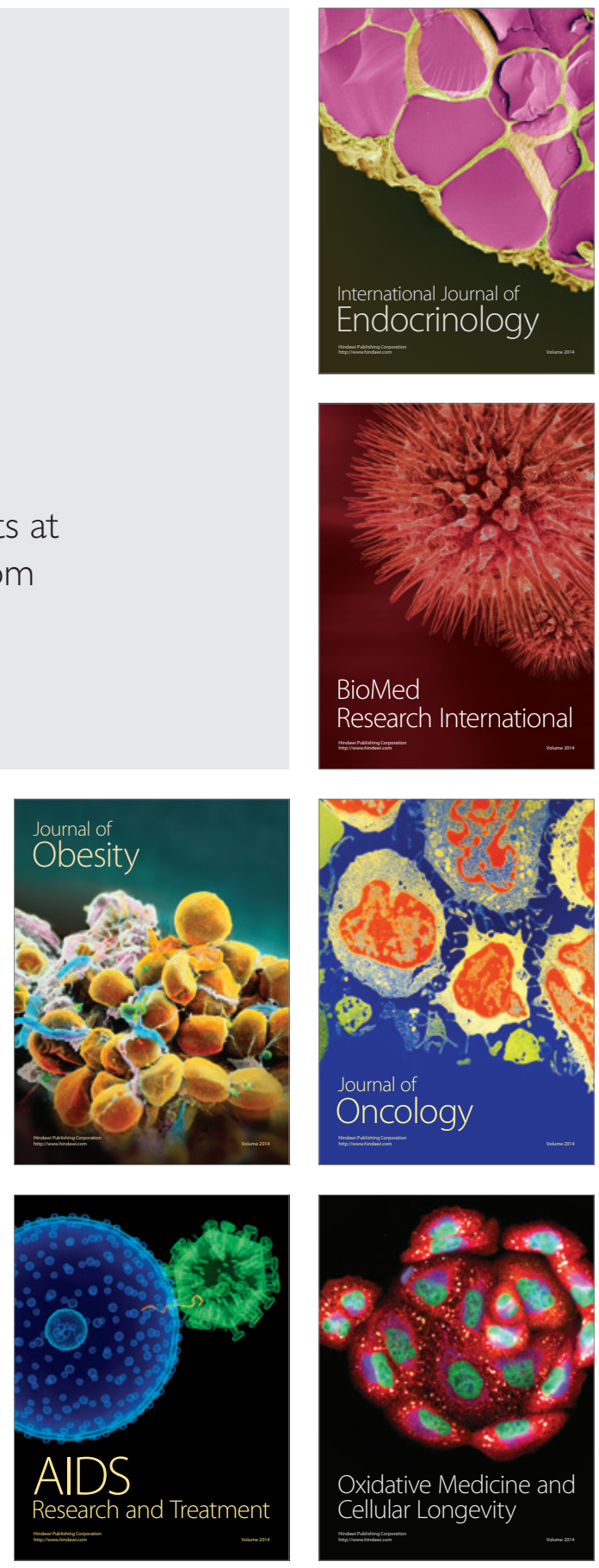